\title{
Référencement Dynamique en Métrologie 3D optique pour une meilleure précision en conditions d'atelier
}

\author{
Jean-François Larue ${ }^{1}$ and Marc Viala ${ }^{2}$ \\ ${ }^{1}$ CREAFORM France SAS, Product Marketing, 24 Rue Jean-Pierre Timbaud, 38600 FONTAINE, France \\ ${ }^{2}$ CREAFORM France SAS, R\&D Department, 24 Rue Jean-Pierre Timbaud, 38600 FONTAINE, France
}

\begin{abstract}
Ce papier décrit les principes du référencement dynamique des MMT optiques et en démontre les avantages en termes de précision de mesure dans les conditions opérationnelles d'une usine. Les récentes MMT à base de triangulation optique offrent la possibilité de mesurer simultanément plusieurs points 3D. Il devient possible, grâce à quelques marqueurs disposés sur la pièce mesurée, de détecter tout déplacement intempestif. Le référentiel machine devient alors identique au référentiel pièce, et reste lié à la pièce durant toute la mesure. Ceci améliore la précision de mesure des MMT portables en présence des vibrations d'un environnement de production.
\end{abstract}

\section{Introduction}

Au cours des 30 dernières années, un des principaux changements en métrologie a été le développement de systèmes de mesure portables, qui a permis d'installer l'inspection au plus près possible de la pièce. Ce changement poussé par le développement des bras de mesure portables au tout début des années 1990 et l'émergence des lasers de poursuite peu après a bouleversé les méthodes d'inspection conventionnelles dans l'industrie. Cela a permis de mesurer plus vite et plus souvent, induisant d'importantes améliorations en temps de réponse et en qualité.

Mais loin du confort des laboratoires de métrologie où des inspecteurs qualifiés utilisent des MMT numériques avec leur marbre stable mais lourd, la mesure portable doit faire face à de nombreux défis. Dans les environnements de production, les vibrations permanentes générées par les équipements de production (ex. : centres d'usinage, presses, équipement de transport, ponts roulants), les changements de température et d'hygrométrie et le niveau de qualification variable des opérateurs sont les obstacles auxquels sont confrontés quotidiennement les utilisateurs.

Les solutions de mesure optique introduisent des concepts innovants tels que le référencement dynamique et l'alignement automatique qui permettent à l'appareil de mesure de rester continuellement verrouillé sur la pièce par un lien optique immatériel. L'objet de l'article est d'évaluer la contribution de ces nouvelles technologies aux opérations de contrôle qualité en environnement usine. L'impact des vibrations sur la précision de mesure sera évalué en s'appuyant sur une présentation des niveaux de vibrations présents dans une usine. L'impact des vibrations sur la précision de la mesure sera quantifié au moyen d'une expérience reproduisant les conditions de l'atelier et s'appuyant sur la norme ASME B89.4.22-2004 [1]. Les principes théoriques mis en œuvre pour le référencement dynamique d'un moyen de mesure optique seront aussi décrits. Enfin des résultats montrant comment l'alignement automatique et le référencement dynamique peuvent influer positivement sur le comportement des métrologues et réduire le risque d'erreurs de mesure seront présentés. Cette contribution se terminera avec une présentation de cas réels issus de l'industrie aéronautique.

\section{Conditions vibratoires rencontrées dans un environnement usine}

\subsection{Les sources de vibrations}

De nombreuses sources de vibrations sont présentes dans un environnement de production en usine :

- proximité de voies routières ou ferrées;

- $\quad$ outils de production (presses, centres

d'usinage);

- $\quad$ appareils de manutention (chariots, grues);

- présence d'opérateurs.

Les vibrations sont transmises au système de mesure et à l'objet mesuré lorsque les planchers de production sont mal isolés des vibrations, et peuvent même être amplifiées par des trépieds instables ou des supports de pièces non rigides.

\subsection{Les niveaux de vibrations}

Des normes définissent des méthodes de quantification des vibrations dans les usines. On citera les normes ISO 2631.2-2003 [2] ou ANSI S3.29-1983 [3]. Il est 
admis que les niveaux de vibrations susceptibles d'entraîner le plus de perturbations correspondent à des plages de vibrations de $1 \mathrm{~Hz}$ à $80 \mathrm{~Hz}$ (les fréquences supérieures sont en partie filtrées par les sols en béton). Les vibrations sont quantifiées sous forme de vitesses ou d'accélérations. Plusieurs études permettent de caractériser les vibrations acceptables pour les travailleurs. Le Department of Environment and Conservation de l'Australie [4] donne ces niveaux en différents endroits dans le tableau 1.

Tableau 1 : Valeurs rms pondérées typiques et maximales de vibrations continues et impulsionnelles $\left(\mathrm{m} / \mathrm{s}^{2}\right) 1-80 \mathrm{~Hz}$

\begin{tabular}{|c|c|c|c|c|c|}
\hline \multirow{2}{*}{ Location } & \multirow{2}{*}{ Assessment period } & \multicolumn{2}{|c|}{ Preferred values } & \multicolumn{2}{|c|}{ Maximum values } \\
\hline & & $z$-axis & $x$-and $y$ & sz-axis & $x$-and $y$-axes \\
\hline \multicolumn{6}{|l|}{ Continous vibration } \\
\hline Critical areas & Day- or night-time & 0.0050 & 0.0036 & 0.010 & 0.0072 \\
\hline \multirow[t]{2}{*}{ Residences } & Daytime & 0.010 & 0.0071 & 0.020 & 0.014 \\
\hline & Night-time & 0.007 & 0.005 & 0.014 & 0.010 \\
\hline $\begin{array}{l}\text { Offices, schools, educationnal } \\
\text { insititutions and places of worship }\end{array}$ & Day- or night-time & 0.020 & 0.014 & 0.040 & 0.028 \\
\hline Workshops & Day-or night-time & 0.04 & 0.029 & 0.080 & 0.058 \\
\hline \multicolumn{6}{|l|}{ Impulsive vibration } \\
\hline Critical areas & Day- or night-time & 0.0050 & 0.0036 & 0.010 & 0.0072 \\
\hline \multirow[t]{2}{*}{ Residences } & Daytime & 0.30 & 0.21 & 0.60 & 0.42 \\
\hline & Night-time & 0.10 & 0.071 & 0.20 & 0.14 \\
\hline $\begin{array}{l}\text { Offices, schools, educationnal } \\
\text { insititutions and places of worship }\end{array}$ & Day- or night-time & 0.64 & 0.46 & 1.28 & 0.92 \\
\hline Workshops & Day-or night-time & 0.64 & 0.46 & 1.28 & 0.92 \\
\hline
\end{tabular}

Une étude [5] présente des enregistrements de vibrations en environnement urbain représentatifs des perturbations générées par des véhicules lourds (autobus, camions). La circulation routière génère dans les bâtiments des niveaux d'accélération entre 0,005 et $2 \mathrm{~m} / \mathrm{s}^{2}$ et des vitesses entre 0,05 et $25 \mathrm{~mm} / \mathrm{s}$ pour des fréquences de 5 à $25 \mathrm{~Hz}$.

Une autre étude [6] propose des enregistrements effectués sur des sites de production. Cette étude réalisée préalablement à l'implantation d'une MMT dans une usine où opèrent des presses d'emboutissage montre des vibrations atteignant 1,06 pouce/sec $(26,9 \mathrm{~mm} / \mathrm{s})$ avec une fréquence de $17 \mathrm{~Hz}$ à 15 mètres de distance de la presse.

Tableau 2 : prédictions de vibrations du sol (vitesse instantanée fonction du temps) induites par des presses à l'emplacement prévisionnel d'une MMT.

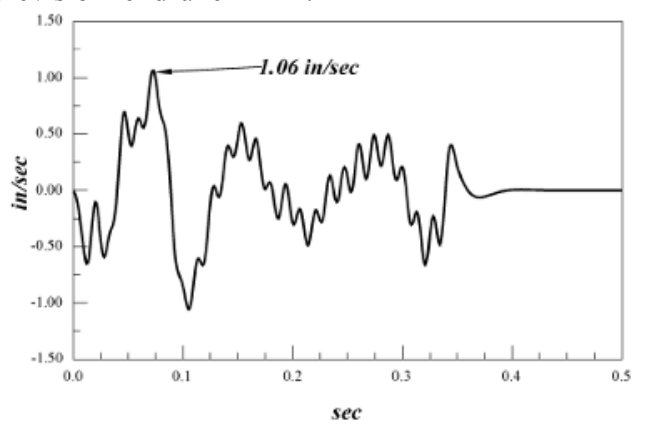

Une dernière étude [7] réalisée en France par l'INRS (Instititut national de recherche et de sécurité) donne des valeurs relevées auprès de machines industrielles :

- $\quad$ Presses à mouler en fonderie : 0,2 à $0,5 \mathrm{~m} / \mathrm{s}^{2}$

- Presses d'emboutissage : 0,25 à $1,15 \mathrm{~m} / \mathrm{s}^{2}$

- Marteau pilon : 2 à $60 \mathrm{~m} / \mathrm{s}^{2}$

On peut retenir de ces études que des accélérations jusqu'à $2 \mathrm{~m} / \mathrm{s}^{2}$ et des vitesses jusqu'à $25 \mathrm{~mm} / \mathrm{s}$ en impulsionnel correspondent à des valeurs courantes dans un atelier de production. Des valeurs d'accélérations de $0,2 \mathrm{~m} / \mathrm{s}^{2}$ ou des vitesses de $2,5 \mathrm{~mm} / \mathrm{s}$ en continu sont également fréquentes dans tout environnement de travail. C'est ce niveau de vibrations que nous avons essayé de reproduire par la suite de façon expérimentale.

\subsection{Le dispositif expérimental}

Nous avons pour cela utilisé un robot ABB IRB 4400 que nous avons programmé pour exécuter des allersretours verticaux de petite amplitude et aussi rapides que possible. On est ici aux limites de l'asservissement du robot. Les déplacements ont été mesurés par un moyen optique.

Tableau 3 : amplitude vibratoire à l'extrémité du robot déplacement vertical en $\mathrm{mm}$ fonction du temps en s)

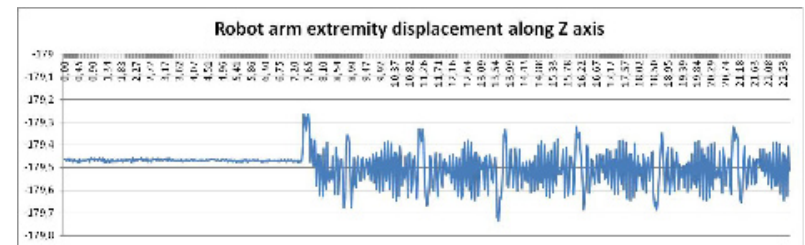

On observe des déplacements d'amplitude crête-crête de $0,4 \mathrm{~mm}$, des accélérations efficaces de l'ordre de 0,25 $\mathrm{m} / \mathrm{s}^{2}$ et des vitesses de $3,4 \mathrm{~mm} / \mathrm{s}$. L'analyse fréquentielle montre une fréquence principale aux alentours de $10 \mathrm{~Hz}$. Ces résultats sont proches de l'objectif visé.

\section{Le référencement dynamique}

\subsection{Les MMT optiques}

Les MMT optiques basées sur la triangulation à partir d'images comprennent un système de localisation optique équipé de caméras vidéo. Deux catégories existent : celles basées sur des caméras matricielles (qui utilisent des cibles rétro-réfléchissantes ou des DEL comme cibles) et celles basées sur des caméras linéaires (qui utilisent toutes des DEL comme cibles). Nous nous intéressons ici uniquement à la première catégorie. Les MMT optiques s'appuient sur trois étapes de calcul importantes:

une étape de traitement d'images pour évaluer précisément les projections des cibles dans les images du capteur stéréo;

- $\quad$ une étape de triangulation permettant d'estimer les coordonnées $(\mathrm{X}, \mathrm{Y}, \mathrm{Z})$ des cibles dans le référentiel capteur à partir de leurs projections dans les deuX images du capteur stéréo;

- $\quad$ une étape d'estimation de la pose d'un objet modélisé par un ensemble de points dont les coordonnées nominales sont connues. 
Figure 1 : principes de fonctionnement d'une MMT optique basée sur la triangulation à partir d'images.

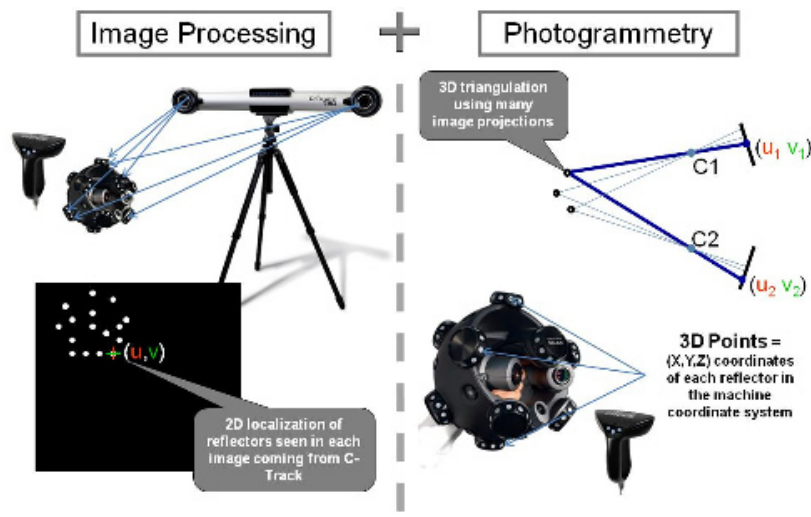

Les caméras se modélisent à l'aide d'une projection perspective et de paramètres additionnels prenant en compte les aberrations géométriques de l'imageur (caméra avec son objectif). L'identification de ces paramètres dits intrinsèques est réalisée lors d'une étape d'étalonnage préalable utilisant des techniques similaires à celles des photogrammètres [8]. Moyennant cet étalonnage, la projection dans un des imageurs d'un point 3D $\mathcal{P}$ exprimé dans le référentiel attaché au capteur stéréo est donnée par la relation de projection perspective [9][10] :

$$
\boldsymbol{x}=\boldsymbol{P} \boldsymbol{X}
$$

Où $\boldsymbol{X}=(X, Y, Z, 1)^{t} \quad$ représente les coordonnées homogènes du point $\mathcal{P} ; \boldsymbol{x}=(u, v, 1)^{t}$ les coordonnées de la projection et $\boldsymbol{P}$ la matrice de projection :

$$
\boldsymbol{P}=\left(\begin{array}{l}
\boldsymbol{P}_{1} \\
\boldsymbol{P}_{2} \\
\boldsymbol{P}_{3}
\end{array}\right)=\boldsymbol{K} \times\left(\begin{array}{c|c}
\boldsymbol{R} & \boldsymbol{t} \\
\mathbf{0}_{1 \times 3} & 1
\end{array}\right)
$$

$\boldsymbol{K}$ étant la matrice de la caméra intégrant ses paramètres intrinsèques (nous faisons abstraction des paramètres de correction des distorsions radiales et tangentielles) :

$$
\boldsymbol{K}=\left(\begin{array}{ccc}
A_{u} & 0 & C_{u} \\
0 & A_{v} & C_{v} \\
0 & 0 & 1
\end{array}\right)
$$

$\boldsymbol{R}$ et $\boldsymbol{t}$ correspondent à la transformation rigide : matrice de rotation et vecteur de translation assurant le passage du référentiel attaché au capteur au référentiel attaché à l'imageur. Cette relation est à la base du calcul des coordonnées d'un point $\mathcal{P}$ par triangulation et du calcul de pose d'un objet. L'étape de traitement d'images consiste à extraire les projections des centres des cibles des images acquises par le capteur stéréo. Dans la littérature, nous trouvons plusieurs approches [11], [12] et [13]. Certaines s'appuient sur le calcul du barycentre des niveaux de gris de la réponse lumineuse de la cible ; les autres sur l'approximation par une ellipse des contours de cette réponse et les dernières par la modélisation de cette réponse. Pour ces dernières techniques, l'estimation du modèle repose sur un estimateur au sens des moindres carrés entre la valeur du pixel mesurée dans l'image $I$ et la valeur du pixel déterminée par le modèle $\hat{I}\left(\boldsymbol{p}_{\text {model }}\right)$ :

$$
\mathcal{C}_{\text {min }}\left(\boldsymbol{p}_{\text {model }}\right)=\sum\left(\hat{I}\left(\boldsymbol{p}_{\text {model }}\right)-I\right)^{2}
$$

L'étape photogrammétrique qui suit la détection souspixellique des cibles est la triangulation: cette étape permet à partir de la mesure des traces d'une cible dans les deux images du capteur stéréo de calculer ses coordonnées $(X, Y, Z)$ dans le référentiel capteur. De nombreuses techniques sont disponibles [8], [14]. La plus simple consiste à prendre le point milieu de la droite perpendiculaire joignant les deux rayons optiques passant par les traces et la cible (Figure).

Figure 2: Principe de la triangulation point milieu

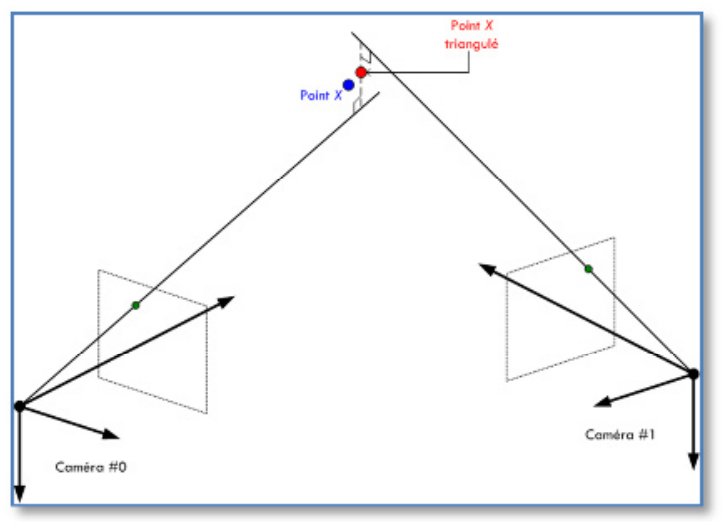

D'autres s'appuient sur la résolution d'un système linéaire à partir de relations de projection perspective (1) et (2) ou d'équations polynomiales. À l'issue de la triangulation, les cibles sont identifiées comme appartenant ou non à un corps rigide. Un corps rigide se décrit comme un ensemble de cibles rigidement liées dans un référentiel propre. Cette identification est suivie d'une localisation $3 \mathrm{D}$ des corps rigides identifiés à partir de la mesure de leurs cibles en 3D et de leurs coordonnées nominales. La localisation 3D permet d'estimer la position et l'orientation du référentiel propre d'un corps rigide, ou pose, par rapport au référentiel de mesure. Une fois la localisation $3 \mathrm{D}$ du corps rigide estimée, la position du centre du palpeur en est déduite. Cela fonctionnera de la même façon si la pointe de touche est remplacée par un capteur constitué d'une projection de ligne laser et de caméra (scanneur).

\subsection{Le référencement dynamique}

Les caméras du système de localisation optique sont capables d'observer simultanément d'autres cibles que celles présentes sur le palpeur. Il est donc possible de disposer des cibles sur l'objet que l'on veut mesurer. Ces cibles étant mesurées en même temps que les cibles du palpeur, la position de la pièce au moment où un point a été palpé peut être calculée. Les caméras étant des caméras matricielles, l'ensemble des cibles est triangulé de façon strictement synchrone à une fréquence de $30 \mathrm{~Hz}$ (certains localisateurs optiques font appel à des cameras linéaires qui ne peuvent acquérir les cibles que de façon séquentielle). Il est donc possible de calculer la position $\mathrm{du}$ palpeur non plus par rapport au référentiel de la machine, mais directement par rapport à la pièce. Toutes les mesures vont ainsi être effectuées dans le référentielpièce et les déplacements éventuels, même rapides, de la 
pièce et du système de localisation optique n'affecteront pas la mesure. Cette fonctionnalité appelée référencement dynamique est au cœur du concept TRUaccuracy des appareils de mesure Creaform.

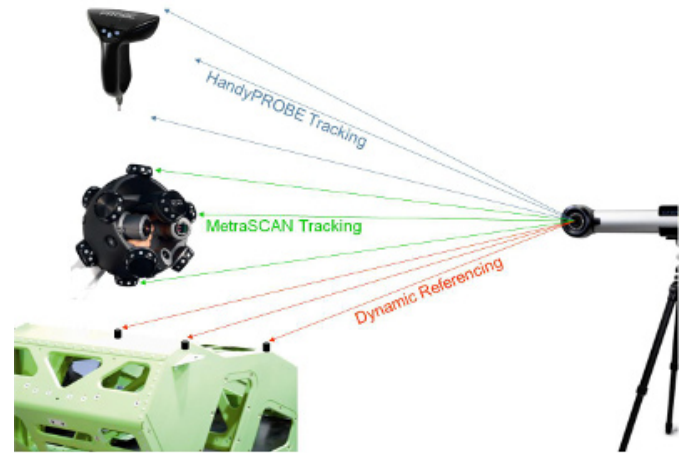

Il est possible de placer la MMT optique sur un trépied léger offrant ainsi une portabilité sans égal puisqu'elle peut être facilement déplacée autour de la pièce pour faciliter la mesure de certains points. Le volume de mesure peut aussi être agrandi sans perdre l'alignement. Le niveau d'incertitude habituellement réservé aux laboratoires de métrologie peut ainsi être atteint dans un environnement perturbé par de nombreuses vibrations.

\section{Résultats expérimentaux}

Une évaluation en laboratoire de l'impact des vibrations sur une MMT portable à bras polyarticulé de 2,40 m et une MMT portable optique a été réalisée. Pour démontrer les performances du référencement dynamique le montage décrit précédemment a été utilisé. Les caractéristiques de charge du robot le

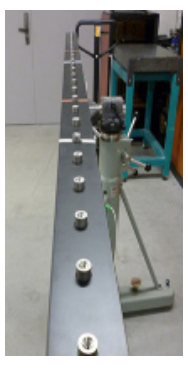
permettant, la machine de mesure portable (respectivement le bras polyarticulé puis le système de localisation optique) a été fixée à l'extrémité du bras du robot. Des essais de précision ont été effectués au moyen d'une règle étalon à cônes de 2,50 $\mathrm{m}$ utilisée pour faire des tests de précision suivant la norme VDI 2634 [15]. La règle était fixée sur un trépied lourd (Fig. 4) L'incertitude d'étalonnage sur les distances est de 1,5 $\mu \mathrm{m}$ $+1 \mu \mathrm{m} \times \mathrm{L}$ avec un facteur d'élargissement $k=2$. Afin d'assurer le référencement dynamique de la MMT optique, des cibles rétro-réfléchissantes avaient été préalablement disposées sur l'ensemble poutre et pied. Vingt-et-un points ont été mesurés sur la barre sur une distance de $2 \mathrm{~m}$ et les distances entre points consécutifs ont été comparées à la distance étalonnée donnée par le certificat d'étalonnage de la règle. Cela donne une erreur de justesse pour chacune des distances considérées (20 distances). Plusieurs mesures ont été effectuées, d'abord deux sans vibrations (robot sur ses freins) puis deux avec les vibrations décrites précédemment (robot en fonctionnement). Les courbes ci-après présentent les résultats obtenus avec les deux moyens de mesure étudiés.
Figure 1 : résultats des tests en l'absence de vibrations pour chaque moyen testé (2 essais). En ordonnée l'écart sur chaque distance entre valeur étalonnée et mesurée.

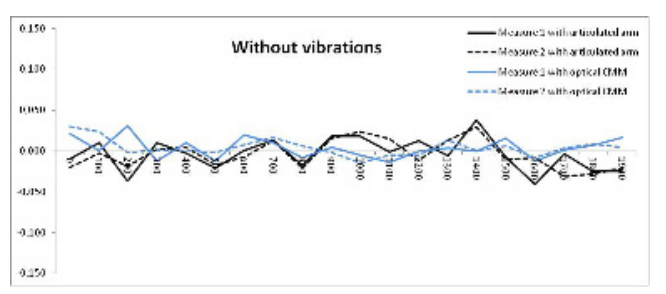

En l'absence de vibrations, les résultats obtenus sont similaires avec une erreur RMS pour le bras de 0,018 $\mathrm{mm}$ et pour la MMT optique de 0,011 mm. Les erreurs maximum sont de $0,041 \mathrm{~mm}$ pour le bras et de 0,031 pour la MMT optique.

Figure 2 : résultats des tests en présence de vibrations pour chaque moyen testé ( 2 essais). En ordonnée l'écart sur chaque distance entre valeur étalonnée et mesurée.

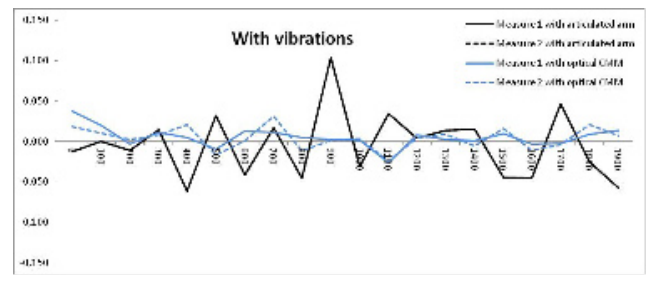

En présence de vibrations, les résultats obtenus montrent clairement le bénéfice du référencement dynamique. Ainsi, on observe une erreur rms de $0,039 \mathrm{~mm}$ pour le bras contre $0,013 \mathrm{~mm}$ pour la MMT optique. Les erreurs maximum montent jusqu'à $0,103 \mathrm{~mm}$ pour le bras tandis qu'elles ne dépassent pas 0,037 pour la MMT optique. On n'observe pas de dégradation notable des performances dans le cas dans la MMT optique.

Rappelons que le niveau de vibrations simulé est très courant dans un environnement atelier. Cette expérience met donc en évidence la perte de précision des solutions portables non optiques en l'absence d'un marbre équipé d'un système de filtrage des vibrations.

\section{Impact sur la réduction des erreurs liées à l'opérateur}

L'étude Measurement Study Report menée en 2011 dans le cadre de CMSC [16] présente une analyse très complète du comportement des opérateurs dans un processus de métrologie.

En particulier, l'étude indique que seulement $6 \%$ des participants du test avec un bras de mesure ont identifié que l'appareil était posé sur de la moquette. De même, seulement $6 \%$ des participants se sont préoccupés de la stabilité de la pièce lors du test avec le laser de poursuite. Dans ce même test, l'analyse établit que $7 \%$ des participants ont déplacé la pièce après avoir effectué l'alignement et que $7 \%$ seulement des participants ont testé une dérive en mesurant de nouveau un point d'alignement à la fin de la séquence. Lors du test avec un laser de poursuite sur un véhicule complet, seulement 
$20 \%$ des participants se sont préoccupés de la présence de moquette, $15 \%$ se sont interrogés sur la stabilité de la pièce, tandis que seulement $25 \%$ ont identifié la nécessité d'avoir un bon alignement.

Plus de $40 \%$ des participants travaillaient dans le domaine du contrôle qualité ou de l'inspection, plus de $60 \%$ avaient 7 ans d'expérience ou plus et plus de $60 \%$ pratiquaient la mesure de façon quotidienne ou hebdomadaire.

Des erreurs de mesures très importantes ont été relevées : « Engine compartment »: de 0,56 à 3,81 mm;

« Door» : de 35 à 43,18 mm;

«Vehicle» : de 5,36 à 8,198 mm.

Il est impossible de dire quelle est la contribution de l'instabilité du montage de mesure à ces erreurs, mais il est possible que cela ait y contribué de façon significative. Une des conclusions de l'étude est que l'erreur humaine est une source majeure de mauvaise qualité de mesure. Le référencement dynamique va contribuer activement à la réduction de certaines erreurs humaines identifiées dans l'étude CMSC, à savoir la mauvaise appréciation par l'opérateur des risques liés à un environnement instable, perturbé par la présence d'un trafic important, ou à une mauvaise stabilité de la pièce.

\section{Exemples d'applications}

\subsection{Mise en œuvre de l'alignement automatique pour minimiser les erreurs opérateurs}

Le référencement dynamique intervient sur un autre paramètre identifié dans l'étude: la qualité de l'alignement. Avec une MMT optique, il est possible au moyen d'outillages spécifiques de positionner certaines cibles sur des points qui doivent servir à l'alignement. Il est alors possible de les mesurer pour positionner automatiquement la MMT optique dans le référentielpièce. On élimine ainsi toutes les erreurs de l'opérateur durant la phase d'alignement et on garantit le meilleur alignement, quel que soit l'opérateur.

Figure 3 : exemple de gabarit réalisé pour automatiser l'alignement et fiabiliser la mesure de pièces aéronautiques

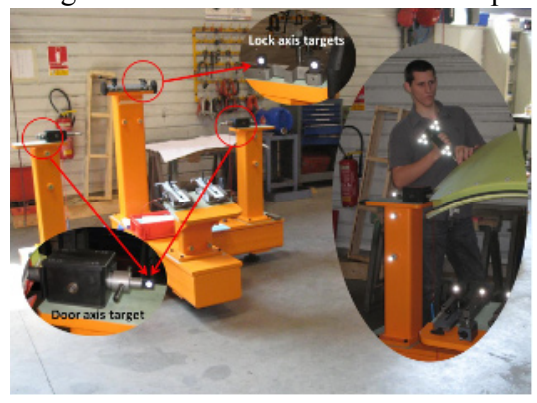

La photo (Figure 3) montre un exemple de gabarit développé pour le contrôle de portes d'inverseurs de poussée des moteurs d'Airbus. Certaines cibles positionnées à des points précis (axe de rotation de la porte, plan d'appui du galet de verrouillage) vont permettre d'aligner automatiquement la porte. Autre avantage, des cibles placées à des emplacements précis sur le gabarit vont assurer le référencement dynamique et permettre de vérifier en temps réel la justesse du moyen de mesure. En effet, en cas de changement de géométrie du référentiel optique ou de dérive de l'appareil, la mesure deviendra impossible indiquant à l'opérateur soit une déformation du gabarit, soit une dérive du moyen de mesure.

\subsection{Mise en place d'un modèle de positionnement dynamique pour la mesure de grandes pièces}

Les cibles rétro-réfléchissantes utilisées pour créer le référentiel dynamique sont également compatibles avec les solutions de photogrammétrie. Il est ainsi possible de créer un modèle de positionnement complet et précis sur la base de cibles disposées sur un objet. Une fois ce modèle complété, il suffit de disposer la MMT optique à un endroit où un nombre suffisant de cibles sont visibles et elle sera automatiquement rigidement liée au référentiel global, ce qui est très intéressant pour mesurer des objets de grande taille. Ainsi, dans le cadre d'un projet avec la société Suisse Jet Aviation AG (une des principales entreprises dans le monde à offrir des services à l'industrie de l'aviation d'affaires), les Services d'ingénierie 3D de Creaform ont eu l'occasion de mesurer et de numériser l'intérieur complet d'un avion de type Boeing 737-800.

L'objectif était d'obtenir une représentation de la géométrie détaillée de l'intérieur de l'appareil permettant à Jet Aviation de concevoir et de fabriquer des aménagements intérieurs sur mesure. Disposer d'un modèle 3D précis permet aux ingénieurs d'identifier à l'avance les risques d'interférences entre les équipements et d'assurer $\mathrm{du}$ premier coup la compatibilité de ceux-ci avec les structures et les systèmes de l'avion.

Figure 4 : Utilisation simultanée de plusieurs appareils dans un environnement instable grâce à un modèle de positionnement dynamique et résultat avec le modèle $3 \mathrm{D}$ reconstruit du fuselage.
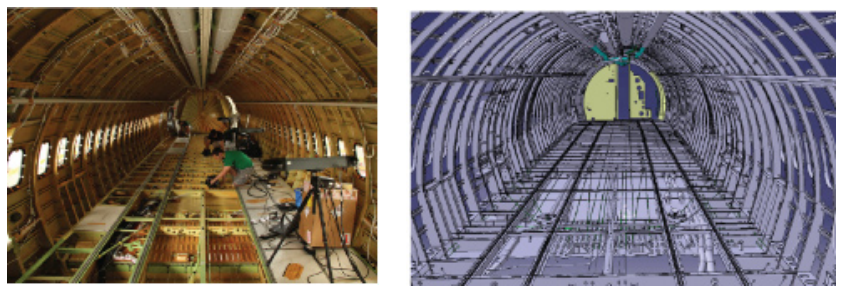

Plusieurs scanneurs à main Handyscan 3D [17], MMT optiques HandyPROBE [18] et scanneurs sur MMT optique MetraSCAN 3D [19] ainsi qu'un système de photogrammétrie MaxSHOT 3D [20] et un scanneur de longue portée Leica ont été utilisés simultanément sans avoir à réaligner les données et surtout, sans avoir à se préoccuper des vibrations provoquées par l'intense activité à l'intérieur de l'appareil. 


\section{Conclusion}

Les MMT optiques portables ouvrent un nouveau chapitre de la mesure 3D. Leurs capacités permettent de rendre la mesure plus fiable, moins dépendante de l'opérateur, encore plus portable. Cela permet surtout de placer la mesure au plus près de la ligne de production, en environnement usine, de détecter rapidement les dérives et d'en déterminer au plus vite les causes, assurant ainsi une meilleure réactivité lors de la montée en puissance de la production.

\section{Références}

1. American Society of Mechanical Engineers (2005). ASME B89.4.22-2004 Method for Performance Evaluation of Articulated Arm Coordinate Measuring Machine. ASME.

2. ISO 2631-2:2003 Mechanical vibration and shock, Evaluation of human exposure to whole-body vibration Part 2: Vibration in buildings $(1 \mathrm{~Hz}$ to $80 \mathrm{~Hz}$ ). ISO.

3. American National Standards Institute. (2006). ANSI s2.71-1983 (r2006) (formerly ANSI s3.29-1983) Guide to the Evaluation of Human Exposure to Vibration in Buildings. ANSI.

4. Australian Government - Department of Environment and Conservation. (2006). Assessing vibrations: a technical guideline.

5. Hunaidi, O. (2000). Réhabilitation des infrastructures urbaines. Montreal: Canadian National research Council. International Organization for Standardization. (2003).

6. Sharif, M. P., \& Warner, B. C. (2000). Prediction of ground vibration induced by operation of heavy machinery. IMAC XX A Conference and Exposition on Structural Dynamics, Conference Proceedings. SPIE Press.
$7 . \quad$ Mistrot, P., Donati, P., \& Galmiche, J. (2e trimestre 1997). Exposition Vibratoire des opérateurs à proximité des machines industrielles. Cahiers de notes documentaires - Hygiène et sécurité du travail n 167 , INRS, Service Métrologie - Acoustique - Vibrations.

8. Atkinson, K. (1996). Close Range Photogrammetry and Machine Vision. Whitlles Publishing.

9. Faugeras, O. (1993). Three-Dimensional Computer Vision (éd. MIT Press). MIT Press

10. Hartley, R., \& Zisserman, A. (2004). Multiple View Geometry in Computer Vision. Cambridge University Press.

11. Shortis, M. R., \& Clarke, T. A. (1994). A comparison of some techniques for the subpixel location of discrete target images. (SPIE, Éd.) Videometrics III, 2350, pp. 239-250.

12. Shortis, M. R., Clarke, T. A., \& Robson, S. (1995). Practical Testing of the Precision and Accuracy of Target Image Centring Algorithms. Videometrics IV, 2598, pp. 65-76.

13. Otepka, J. (2004). Precision Target Mensuration in Vision Metrology. Vienne: Technische Universität Wien.

14. Hartley, R. I., \& Sturm, P. (1997, Novembre). Triangulation. Computer Vision and Image Understanding , 68 (2), pp. 146-157.

15. Verein Deutscher Ingenieure. (2002). VDI/VDE 2634 part 1 : Optical 3D measuring systems - Imaging systems with point-by-point probing.

16. Bevan, K., \& Toman, T. (2011). How Behavior Impacts Your Measurement. CMSC 2011 Measurement Study Report.

17. Creaform 3D. (s.d.). Handyscan 3D scanners - Handheld3D scanners. (Creaform 3D) sur

www.creaform3d.com/en/handyscan3d/produits/default

18. Creaform 3D. (s.d.). HandyPROBE - Portable coordinate measuring machine sur www.creaform3d.com/en/handyprobe/handyprobe/default

19. Creaform3D MetraSCAN optical CMM scanner sur www.creaform 3 d.com/en/metrascan/default.aspx

20. Creaform 3D. (s.d.). MaxSHOT 3D Optical Coordinate Measuring System sur www.creaform3d.com/en/maxshot3d/default.aspx 